\title{
An Improved Parameter Computation Method for a MIMO V2V Rayleigh Fading Channel Simulator Under Non-Isotropic Scattering Environments
}

\author{
Xiang Cheng, Qi Yao, Cheng-Xiang Wang, Bo Ai, Gordon L. Stüber, Dongfeng Yuan, and Bing-Li Jiao
}

\begin{abstract}
For simulations of multiple-input multiple-output (MIMO) vehicle-to-vehicle (V2V) Rayleigh fading channels under more realistic non-isotropic scattering scenarios, we propose a new parameter computation method for the design of sum-ofsinusoids (SoS) simulation models. Compared with the existing relevant methods, the proposed method has the same simulation efficiency but provides a better approximation to the desired statistical properties of the theoretical reference model.
\end{abstract}

Index Terms-SoS simulator, MIMO V2V fading channels, non-isotropic scattering, parameter computation methods.

\section{INTRODUCTION}

A CCURATE and efficient vehicle-to-vehicle (V2V) channel simulation models play a major role in the design and performance evaluation of $\mathrm{V} 2 \mathrm{~V}$ communication systems [1]. Deterministic sum-of-sinusoids ( $\mathrm{SoS}$ ) simulation approach has been widely used in the design of channel simulators due to its high simulation efficiency [2]-[4]. A key issue on the design of deterministic simulation models is how to properly design accurate and efficient parameter computation methods. The investigation of V2V deterministic SoS channel simulators first concentrated on the design of parameter computation methods under isotropic scattering environments [5], [6]. In this case, the modified method of exact Doppler spread (MEDS) has been widely accepted as the most accurate and efficient parameter computation method. However, real V2V

Manuscript received July 12, 2012. The associate editor coordinating the review of this letter and approving it for publication was T. Tsiftsis.

This work was jointly supported by the National Natural Science Foundation of China (Grant no. 61101079 and 61222105), the Science Foundation for the Youth Scholar of Ministry of Education of China (Grant no. 20110001120129), the open research fund of the National Mobile Communications Research Laboratory (Grant no. 2012D06), Southeast University, the RCUK for the UK-China Science Bridges Project: R\&D on (B)4G Wireless Mobile Communications, the Opening Project of the Key Laboratory of Cognitive Radio and Information Processing (Guilin University of Electronic Technology), the Ministry of Education (No.: 2011KF01), and the Beijing Municipal Natural Science Foundation (Grant no. 4112048).

X. Cheng and B.-L. Jiao are with the School of Electronics Engineering \& Computer Science, Peking University, Beijing, China (e-mail: \{xiangcheng, jiaobl\}@pku.edu.cn). X. Cheng is also with the National Mobile Communications Research Laboratory, Southeast University, Nanjing, China.

Q. Yao and C.-X. Wang (corresponding author) are with the School of Information Science and Engineering, Shandong University, Jinan 250100, Shandong, China, and the Joint Research Institute for Signal and Image Processing, School of Engineering \& Physical Sciences, Heriot-Watt University, Edinburgh EH14 4AS, UK (e-mail: qiyao2001 @ hotmail.com; chengxiang.wang@hw.ac.uk).

B. $\mathrm{Ai}$ is with the State Key Laboratory of Rail Traffic Control and Safety, Beijing Jiaotong University, Beijing 100044, China (e-mail: boai@bjtu.edu.cn).

G. Stüber is with the School of Electrical and Computer Engineering, Georgia Institute of Technology, Atlanta, GA 30332 USA (e-mail: stuber@ece.gatech.edu).

D. Yuan is with the School of Information Science and Engineering, Shandong University, Jinan, 250100, China (e-mail: dfyuan@sdu.edu.cn).

Digital Object Identifier 10.1109/LCOMM.2013.011113.121535 propagation scenarios in general do not satisfy the isotropic scattering assumption [7]. Recently, a number of researches focused on the design of $\mathrm{V} 2 \mathrm{~V}$ deterministic SoS channel simulators for non-isotropic scattering channels [8]-[11].

Three parameter computation methods, i.e., the $L_{p}$-norm method (LPNM) [8] and two different modified method of equal areas (MMEA) [9]-[10] based on the MEA [12], have been developed for the design of non-isotropic V2V deterministic SoS channel simulators. Compared with the MMEA, the LPNM depends on numerical optimization techniques and thus has higher computational expenditure. On the other hand, neither of the two MMEAs can meet the accuracyefficiency design criteria that guarantee the designed SoS channel simulator to have the best accuracy-efficiency tradeoff [11]. Therefore, the development of new parameter computation methods that meet the accuracy-efficiency design criteria is desirable to facilitate the design and performance analysis of $\mathrm{V} 2 \mathrm{~V}$ systems for non-isotropic scattering environments.

In this letter, we propose a new parameter computation method, named as improved MMEA (IMMEA), for the design of multiple-input multiple-output (MIMO) V2V deterministic SoS channel simulators under non-isotropic scattering environments. The proposed IMMEA has the ability to meet the accuracy-efficiency design criteria. Simulation results show that compared with the existing MMEAs, the proposed IMMEA provides better approximations to the desired statistical properties without scarifying any simulation efficiency.

\section{The Theoretical Reference Model}

In this letter, a double-bounce two-ring MIMO V2V geometry-based stochastic model is utilized as the theoretical reference model [7]. Suppose there are $N_{1}$ and $N_{2}$ effective scatterers around the transmitter $(\mathrm{Tx})$ and receiver $(\mathrm{Rx})$ lying on two rings of radii $R_{T}$ and $R_{R}$, respectively. The distance between the $\mathrm{Tx}$ and $\mathrm{Rx}$ is $D$. The antenna element spacings at the Tx and $\mathrm{Rx}$ are $\delta_{T}$ and $\delta_{R}$, respectively. The reasonable assumptions $\min \left\{R_{T}, R_{R}\right\}>\max \left\{\delta_{T}, \delta_{R}\right\}$ and $D \gg \max \left\{\delta_{T}, \delta_{R}\right\}$ are used here. The multi-element antenna tilt angles are $\beta_{T}$ and $\beta_{R}$. The Tx and Rx move with speeds $v_{T}$ and $v_{R}$ in directions determined by the angles of motion $\gamma_{T}$ and $\gamma_{R}$, respectively. The angle of arrival (AoA) of the waves traveling from an effective scatterer at the $\mathrm{Rx}$ ring towards the $\mathrm{Rx}$ is denoted by $\phi_{R}^{\left(n_{2}\right)}\left(n_{2}=1, \ldots, N_{2}\right)$ and the angle of departure (AoD) of the waves that impinge on an effective scatterer at the Tx ring is designated by $\phi_{T}^{\left(n_{1}\right)}\left(n_{1}=1, \ldots, N_{1}\right)$.

The MIMO fading channel can be described by an $M_{R} \times$ $M_{T}$ matrix $\mathbf{H}(t)=\left[h_{p q}(t)\right]_{M_{R} \times M_{T}}$, where $h_{p q}(t)$ denotes the complex fading envelope process between the $p$ th $(p=$ $\left.1,2, \ldots, M_{T}\right)$ Tx and the $q$ th $\left(q=1,2, \ldots, M_{R}\right) \mathrm{Rx}$ antenna elements and can be expressed by [7] 
$h_{p q}(t)=\lim _{N_{1}, N_{2} \rightarrow \infty} \sum_{n_{1}, n_{2}=1}^{N_{1}, N_{2}} \frac{e^{j\left[2 \pi f_{T_{m a x}} t \psi_{D}+\psi_{n_{1}, n_{2}}-2 \pi f_{c} \tau_{p q, n_{1}, n_{2}}\right]}}{\sqrt{N_{1} N_{2}}}$

where $\psi_{D}=f_{T_{\max }} \cos \left(\phi_{T}^{\left(n_{1}\right)}-\gamma_{T}\right)+f_{R_{\max }} \cos \left(\phi_{R}^{\left(n_{2}\right)}-\gamma_{R}\right)$, $f_{c}$ is the carrier frequency, $f_{T_{\max }}=v_{T} / \lambda$ and $f_{R_{\max }}=v_{R} / \lambda$ are the maximum Doppler frequencies associated with the Tx and $\mathrm{Rx}$, respectively, and $\lambda=c / f_{c}$ is the carrier wavelength with $c$ denoting the speed of light. The phase $\psi_{n_{1}, n_{2}}$ is a random variable uniformly distributed within $[-\pi, \pi)$. It is assumed that $\phi_{R}^{\left(n_{2}\right)}, \phi_{T}^{\left(n_{1}\right)}$, and $\psi_{n_{1}, n_{2}}$ are mutually independent. In (1), $\tau_{p q, n_{1}, n_{2}}=\left(\varepsilon_{p n_{1}}+\varepsilon_{n_{1} n_{2}}+\varepsilon_{n_{2} q}\right) / c$ is the travel time of the waves from the Tx to the Rx with $\varepsilon_{p n_{1}} \approx$ $R_{T}-k_{p} \delta_{T} \cos \left(\phi_{T}^{\left(n_{1}\right)}-\beta_{T}\right), \varepsilon_{n_{2} q} \approx R_{R}-k_{q} \delta_{R} \cos \left(\phi_{R}^{\left(n_{2}\right)}-\beta_{R}\right)$, and $\varepsilon_{n_{1} n_{2}} \approx D$, where $k_{p}=M_{T}-2 p+1 / 2$ and $k_{q}=M_{R}-2 q+1 / 2$.

Since the numbers $N_{1}$ and $N_{2}$ in (1) tend to infinity, the discrete expressions of the AoA $\phi_{R}^{\left(n_{2}\right)}$ and AoD $\phi_{T}^{\left(n_{1}\right)}$ can be replaced by the continuous expressions $\phi_{R}$ and $\phi_{T}$, respectively. Without loss of generality, the von Mises probability density function (PDF) [13] is utilized to describe the distribution of AoA and AoD. It is defined as $f(\phi) \triangleq \exp [k \cos (\phi-\mu)] /\left[2 \pi I_{0}(k)\right]$, where $\phi \in[-\pi, \pi), I_{0}(\cdot)$ is the zeroth-order modified Bessel function of the first kind, $\mu \in[-\pi, \pi)$ accounts for the mean value of the angle $\phi$, and $k(k \geq 0)$ is a real-valued parameter that controls the angle spread of the angle $\phi$. For $k=0$ (isotropic scattering), the von Mises PDF reduces to the uniform distribution, while for $k>0$ (non-isotropic scattering), the von Mises PDF approximates different distributions based on the values of $k$ [13]. Applying the von Mises PDF to the AoA $\phi_{R}$ and AoD $\phi_{T}$, we obtain $f\left(\phi_{R}\right) \triangleq \exp \left[k_{R} \cos \left(\phi_{R}-\mu_{R}\right)\right] /\left[2 \pi I_{0}\left(k_{R}\right)\right]$ and $f\left(\phi_{T}\right) \triangleq \exp \left[k_{T} \cos \left(\phi_{T}-\mu_{T}\right)\right] /\left[2 \pi I_{0}\left(k_{T}\right)\right]$, respectively.

Under the wide sense stationary (WSS) condition, the spacetime correlation function (STCF) of the reference model can be expressed by [7]

$$
\begin{aligned}
& \rho_{h_{p q} h_{p^{\prime} q^{\prime}}}(\tau)=\mathbf{E}\left[h_{p q}(t) h_{p^{\prime} q^{\prime}}^{*}(t-\tau)\right] \\
& =\frac{I_{0}\left\{\sqrt{\left(A_{T}\right)^{2}+\left(B_{T}\right)^{2}}\right\} I_{0}\left\{\sqrt{\left(A_{R}\right)^{2}+\left(B_{R}\right)^{2}}\right\}}{I_{0}\left(k_{T}\right) I_{0}\left(k_{R}\right)}
\end{aligned}
$$

where $A_{T}=k_{T} \cos \mu_{T}+j 2 \pi \tau f_{T_{\text {max }}} \cos \gamma_{T}+j 2 \pi P \cos \beta_{T}$, $A_{R}=k_{R} \cos \mu_{R}+j 2 \pi \tau f_{R_{\text {max }}} \cos \gamma_{R}+j 2 \pi Q \cos \beta_{R}, B_{T}=$ $k_{T} \sin \mu_{T}+j 2 \pi \tau f_{T_{\max }} \sin \gamma_{T}+j 2 \pi P \sin \beta_{T}, B_{R}=k_{R} \sin \mu_{R}+$ $j 2 \pi \tau f_{R_{\max }} \sin \gamma_{R}+j 2 \pi Q \sin \beta_{R}, \tau$ is the time separation, $(\cdot)^{*}$ denotes the complex conjugate operation, $\mathbf{E}[\cdot]$ is the statistical expectation operator, $p, p^{\prime} \in\left\{1,2, \ldots, M_{T}\right\}, q, q^{\prime} \in$ $\left\{1,2, \ldots, M_{R}\right\}, P=\left(p^{\prime}-p\right) \delta_{T} / \lambda$, and $Q=\left(q^{\prime}-q\right) \delta_{R} / \lambda$.

\section{A New Parameter Computation Method for THE Deterministic SoS Simulation Model}

The above theoretical reference model assumes infinite numbers of effective scatterers and thus cannot be used for simulations. Based on the proposed reference model, this section will design a deterministic SoS simulation model that has reasonable complexity, i.e., finite numbers of effective scatterers. Similar to (1), we can express the complex fading envelope process $\widetilde{h}_{p q}(t)$ of the deterministic SoS simulation model as

$$
\begin{array}{r}
\widetilde{h}_{p q}(t)=\sum_{n_{1}, n_{2}=1}^{N_{1}, N_{2}} \frac{1}{\sqrt{N_{1} N_{2}}} e^{j 2 \pi f_{T_{\text {max }}} t \cos \left(\widetilde{\phi}_{T}^{\left(n_{1}\right)}-\gamma_{T}\right)} \\
\times e^{j\left[2 \pi f_{R_{\text {max }}} t \cos \left(\widetilde{\phi}_{R}^{\left(n_{2}\right)}-\gamma_{R}\right)+\widetilde{\psi}_{n_{1}, n_{2}}-2 \pi f_{c} \tau_{p q, n_{1}, n_{2}}\right]}
\end{array}
$$

where $\widetilde{\phi}_{R}^{\left(n_{2}\right)}$ and $\widetilde{\phi}_{T}^{\left(n_{1}\right)}$ are discrete AoAs and AoDs, respectively, which remain constant for different simulation trials due to the deterministic nature of the simulator. The phase $\widetilde{\psi}_{n_{1}, n_{2}}$ is a single realization of a random generator uniformly distributed over $[-\pi, \pi)$. It is assumed that $\widetilde{\phi}_{R}^{\left(n_{2}\right)}, \widetilde{\phi}_{T}^{\left(n_{1}\right)}$, and $\widetilde{\psi}_{n_{1}, n_{2}}$ are independent.

A key issue on the design of the deterministic SoS simulation model is to find proper sets of AoA $\left\{\widetilde{\phi}_{R}^{\left(n_{2}\right)}\right\}_{n_{2}=1}^{N_{2}}$ and $\operatorname{AoD}\left\{\widetilde{\phi}_{T}^{\left(n_{1}\right)}\right\}_{n_{1}=1}^{N_{1}}$ that make the simulator reproduce the desired statistical properties of the reference model as faithfully as possible with reasonable complexity. To this end, the following two accuracy-efficiency design criteria should be met.

1) Criterion 1: The discrete AoA and AoD should be designed in such a manner that they are distributed within a proper and complete range, in which the $\mathrm{AoA} / \mathrm{AoD}$ carries all the necessary information for the design of a parameter computation method. For isotropic scattering environments, the proper and complete design range is $[0, \pi / 2)$ [5]. For non-isotropic scattering scenarios, the whole range $[-\pi, \pi)$ is necessary for the proper design of the AoA/AoD [11], i.e., $\widetilde{\phi}_{R}^{\left(n_{2}\right)}, \widetilde{\phi}_{T}^{\left(n_{1}\right)} \in[-\pi, \pi)$.

2) Criterion 2: The discrete AoAs/AoDs must be disjoint or unique, i.e., $\widetilde{\phi}_{R}^{\left(n_{2}\right)} \neq \pm \widetilde{\phi}_{R}^{\left(m_{2}\right)}$ with $n_{2} \neq m_{2}$ and $\widetilde{\phi}_{T}^{\left(n_{1}\right)} \neq$ $\pm \widetilde{\phi}_{T}^{\left(m_{1}\right)}$ with $n_{1} \neq m_{1}$, where $n_{2}, m_{2}=1,2, \ldots, N_{2}$ and $n_{1}, m_{1}=1,2, \ldots, N_{1}$.

As mentioned in [11], it is not easy to find the sets of AoA and AoD to meet Criterion 2 for all non-isotropic scattering scenarios. Currently, two available MMEAs [9], [10] cannot solve the difficulty in finding the proper sets of AoA and AoD to meet the aforementioned two accuracy-efficiency design criteria. Therefore, neither of the available MMEAs consistently outperforms the other one for all non-isotropic scattering MIMO V2V scenarios. This motivates us to propose a new parameter computation method, named as IMMEA, to solve this difficulty. For comparison purposes, we first give a brief description of these two existing MMEAs.

\section{A. Existing MMEAs}

Both existing MMEAs originate from the MEA [12], which was designed for isotropic scattering scenarios. The first MMEA was proposed in [9] and is named as MMEA1 in this letter. The MMEA1 is designed to select the set of AoA in such a manner that the areas under the PDF $f\left(\phi_{R}\right)$ of the AoA in different ranges of $\widetilde{\phi}_{R}^{\left(n_{2}-1\right)} \leq \phi_{\sim^{(1)}}<\widetilde{\phi}_{R}^{\left(n_{2}\right)}$ are equal to each other with the initial condition $\int_{\mu_{R}-\pi}^{\widetilde{\phi}_{R}^{(1)}} f\left(\phi_{R}\right) d \phi_{R}=\frac{1-1 / 4}{N_{2}}$. This means that the MMEA1 designs the AoA of the deterministic model as $\frac{n_{2}-1 / 4}{N_{2}}=\int_{\mu_{R}-\pi}^{\widetilde{\phi}_{R}^{\left(n_{2}\right)}} f\left(\phi_{R}\right) d \phi_{R}$, where $n_{2}=$ $1,2, \ldots, N_{2}$ and $\phi_{R} \in[-\pi, \pi)$. Similarly, the AoD can be obtained by using the MMEA1 as $\frac{n_{1}-1 / 4}{N_{1}}=\int_{\mu_{T}-\pi}^{\widetilde{\phi}_{T}^{\left(n_{1}\right)}} f\left(\phi_{T}\right) d \phi_{T}$, where $n_{1}=1,2, \ldots, N_{1}$ and $\phi_{T} \in[-\pi, \pi)$. If the mean angles $\mu_{R} \leq 0$ and $\mu_{T} \leq 0$, we have 


$$
\begin{aligned}
& \widetilde{\phi}_{R}^{\left(n_{2}\right)}=F_{R}^{-1}\left(\frac{n_{2}-1 / 4}{N_{2}}\right), \widetilde{\phi}_{R}^{\left(n_{2}\right)} \in[-\pi, \pi) \\
& \widetilde{\phi}_{T}^{\left(n_{1}\right)}=F_{T}^{-1}\left(\frac{n_{1}-1 / 4}{N_{1}}\right), \widetilde{\phi}_{T}^{\left(n_{1}\right)} \in[-\pi, \pi)
\end{aligned}
$$

where $F_{R}^{-1}(\cdot)$ and $F_{T}^{-1}(\cdot)$ denote the inverse function of the cumulative distribution function $(\mathrm{CDF})$ for $\phi_{R}$ and $\phi_{T}$, respectively. For $\mu_{R}>0$ and $\mu_{T}>0$, it is clear that $\widetilde{\phi}_{R}^{\left(n_{2}\right)}$ and $\widetilde{\phi}_{T}^{\left(n_{1}\right)}$ are designed over the range $\left[\mu_{R}-\pi, \pi\right)$ and $\left[\mu_{T}-\pi, \pi\right)$, respectively, rather than over the whole range $[-\pi, \pi)$, which violates Criterion 1 for non-isotropic scattering environments. Therefore, for $\mu_{R}>0$ and $\mu_{T}>0$, the performance of the MMEA1 should dramatically decrease, which will be demonstrated in Section IV. Note that as mentioned in [11], the MMEA1 can meet the two accuracy-efficiency design criteria only when $\mu_{R}=\mu_{T}=0$.

The other MMEA was proposed in [10] and we name it as MMEA2, to distinguish from the MMEA1. The MMEA2 designs the AoA in such a manner that the areas under the PDF $f\left(\phi_{R}\right)$ of the AoA in different ranges of $\widetilde{\phi}_{R}^{\left(n_{2}-1\right)} \leq$ $\phi_{R}<\underset{\widetilde{\phi}_{R}^{(1)}}{\widetilde{\phi}_{R}^{\left(n_{2}\right)}}$ are equal to each other with the initial condition $\int_{-\pi}^{\widetilde{\phi}_{R}^{(1)}} f\left(\phi_{R}\right) d \phi_{R}=\frac{1-1 / 2}{N_{2}}$. Therefore, the AoA of the deterministic simulator is designed as

$$
\widetilde{\phi}_{R}^{\left(n_{2}\right)}=F_{R}^{-1}\left(\frac{n_{2}-1 / 2}{N_{2}}\right), \widetilde{\phi}_{R}^{\left(n_{2}\right)} \in[-\pi, \pi) .
$$

Similarly, the MMEA2 designs the AoD as

$$
\widetilde{\phi}_{T}^{\left(n_{1}\right)}=F_{T}^{-1}\left(\frac{n_{1}-1 / 2}{N_{1}}\right), \widetilde{\phi}_{T}^{\left(n_{1}\right)} \in[-\pi, \pi) .
$$

It is obvious that the MMEA2 chooses the value of $1 / 2$ instead of $1 / 4$ in the MMEA1. This is seemly inspired by the MEDS in [5] for the simulation of isotropic scattering V2V channels. However, the MMEA2 cannot meet Criterion 2 for all nonisotropic scattering MIMO V2V scenarios.

\section{B. A New Parameter Computation Method - IMMEA}

In the following, we will derive the IMMEA that has the ability to meet the two accuracy-efficiency design criteria for any non-isotropic scattering MIMO V2V channels. Taking the design of the AoA $\widetilde{\phi}_{R}^{\left(n_{2}\right)}$ as an example, the IMMEA includes the following three steps:

1) Define a new random variable $\phi_{R}^{\prime} \in\left[\mu_{R}-\pi, \mu_{R}+\pi\right)$. It follows a von Mises distribution having the same parameters, i.e., $\mu_{R}$ and $k_{R}$, as $\phi_{R} \in[-\pi, \pi)$.

2) Then, we design the proper set of $\left\{\widetilde{\phi}_{R}^{\prime\left(n_{2}\right)}\right\}_{n_{2}=1}^{N_{2}}$ as

$\widetilde{\phi}_{R}^{\prime\left(n_{2}\right)}=F_{R}^{\prime-1}\left(\frac{n_{2}-1 / 4}{N_{2}}\right), \widetilde{\phi}_{R}^{\prime\left(n_{2}\right)} \in\left[\mu_{R}-\pi, \mu_{R}+\pi\right)$

where $F_{R}^{\prime-1}(\cdot)$ denotes the inverse function of the von Mises CDF for $\phi_{R}^{\prime}$.

3) Obtain the desired set of $\left\{\widetilde{\phi}_{R}^{\left(n_{2}\right)}\right\}_{n_{2}=1}^{N_{2}}$ by properly mapping $\widetilde{\phi}_{R}^{\prime\left(n_{2}\right)}$ to the range of $[-\pi, \pi)$ :

$$
\widetilde{\phi}_{R}^{\left(n_{2}\right)}= \begin{cases}\widetilde{\phi}_{R}^{\prime\left(n_{2}\right)}+2 \pi, & \text { if } \widetilde{\phi}_{R}^{\prime\left(n_{2}\right)}<-\pi \\ \widetilde{\phi}_{R}^{\prime\left(n_{2}\right)}-2 \pi, & \text { if } \widetilde{\phi}_{R}^{\prime\left(n_{2}\right)} \geq \pi \\ \widetilde{\phi}_{R}^{\prime\left(n_{2}\right)}, & \text { else. }\end{cases}
$$

Similarly, the AoD can be designed by following the above 3 steps of the IMMEA. First, we define a random variable $\phi_{T}^{\prime}$ over the range $\left[\mu_{T}-\pi, \mu_{T}+\pi\right)$ fulfilling the von Mises distribution with the same mean angle $\mu_{T}$ and parameter $k_{T}$ as the random variable $\phi_{T}$. The discrete realizations of the new defined random variable $\phi_{T}^{\prime\left(n_{l}\right)}$ can then be designed as

$$
\widetilde{\phi}_{T}^{\prime\left(n_{1}\right)}=F_{T}^{\prime-1}\left(\frac{n_{1}-1 / 4}{N_{1}}\right), \widetilde{\phi}_{T}^{\prime\left(n_{1}\right)} \in\left[\mu_{T}-\pi, \mu_{T}+\pi\right)
$$

where $F_{T}^{\prime-1}(\cdot)$ denotes the inverse function of the von Mises $\mathrm{CDF}$ for $\phi_{T}^{\prime}$. The AoD of the simulation model can be obtained by mapping $\widetilde{\phi}_{T}^{\prime\left(n_{l}\right)}$ to the range of $[-\pi, \pi)$ as

$$
\widetilde{\phi}_{T}^{\left(n_{1}\right)}= \begin{cases}\widetilde{\phi}_{T}^{\prime\left(n_{1}\right)}+2 \pi, & \text { if } \widetilde{\phi}_{T}^{\left(n_{1}\right)}<-\pi \\ \widetilde{\phi}_{T}^{\prime\left(n_{1}\right)}-2 \pi, & \text { if } \widetilde{\phi}_{T}^{\prime\left(n_{1}\right)} \geq \pi \\ \widetilde{\phi}_{T}^{\prime\left(n_{1}\right)}, & \text { else. }\end{cases}
$$

It is clear that the proposed IMMEA will reduce to the MMEA1 when $\mu_{R}=\mu_{T}=0$ holds. Note that these three different MMEAs have completely different selected sets of AoA/AoD and thus express different simulation accuracy. Among them, only the proposed IMMEA can meet the accuracy-efficiency design criteria for any non-isotropic scattering MIMO V2V environments, i.e., for different von Mises distributions with any values of $\mu_{R}, \mu_{T}, k_{R}$, and $k_{T}$. Therefore, the IMMEA should outperform the MMEA1 and MMEA2, which will be demonstrated in Section IV.

Due to the deterministic nature of the proposed simulator, its STCF must be analyzed by using time averages rather than statistical averages, i.e., $\widetilde{\rho}_{\widetilde{h}_{p q} \widetilde{h}_{p^{\prime} q^{\prime}}}(\tau)=\left\langle\tilde{h}_{p q}(t) \widetilde{h}_{p^{\prime} q^{\prime}}^{*}(t-\tau)\right\rangle$, where $\langle\cdot\rangle$ denotes the time average operator. Substituting (3) into the above defined STCF and following the similar derivation procedure in [11], we have

$$
\begin{gathered}
\widetilde{\rho}_{\widetilde{h}_{p q} \widetilde{h}_{p^{\prime} q^{\prime}}}(\tau)=\frac{1}{2 N_{1} N_{2}} \sum_{n_{1}, n_{2}=1}^{N_{1}, N_{2}} \frac{2 \pi f_{c}}{e^{c}}\left[\delta_{T} \cos \left(\widetilde{\phi}_{T}^{\left(n_{1}\right)} \beta_{T}\right)+\delta_{R} \cos \left(\widetilde{\phi}_{R}^{\left(n_{2}\right)}-\beta_{R}\right)\right] \\
\times e^{\left[2 \pi f_{T_{\max }} \tau \cos \left(\widetilde{\phi}_{T}^{\left(n_{1}\right)}-\gamma_{T}\right)+2 \pi f_{R_{\max }} \tau \cos \left(\widetilde{\phi}_{R}^{\left(n_{2}\right)}-\gamma_{R}\right)\right]}
\end{gathered}
$$

It can be shown that for $\left\{N_{1}, N_{2}\right\} \rightarrow \infty$, the STCF of the deterministic simulation model tends to that of the reference model, i.e., $\lim _{N_{1}, N_{2} \rightarrow \infty} \widetilde{\rho}_{\widetilde{h}_{p q}} \widetilde{h}_{p^{\prime} q^{\prime}}(\tau)=\rho_{h_{p q} h_{p^{\prime} q^{\prime}}}(\tau)$ holds.

\section{NumericAl Results AND ANALYSis}

In this section, we investigate the performance of the proposed IMMEA-based deterministic SoS simulation model in terms of its STCF. Unless otherwise specified, all the results presented in this section were obtained using $f_{c}=5.9 \mathrm{GHz}$, $f_{T_{\max }}=f_{R_{\max }}=570 \mathrm{~Hz}, \tau_{\max }=5 / f_{T_{\max }}, \beta_{T}=\beta_{R}=\gamma_{T}=$ $\gamma_{R}=50^{\circ}, D=300 \mathrm{~m}, R_{T}=R_{R}=12 \mathrm{~m}, k_{T}=k_{R}=1$, and $N_{1}=N_{2}=30$ for the deterministic channel simulator.

Figs. 1 (a) and (b) present a comparison of the squared error $\varepsilon$ of the STCF, defined as $\varepsilon=\left|\widetilde{\rho}_{\widetilde{h}_{p q}} \widetilde{h}_{p^{\prime} q^{\prime}}(\tau)-\rho_{h_{p q} h_{p^{\prime} q^{\prime}}}(\tau)\right|^{2}$, for the deterministic simulation model using the MMEA1, MMEA2, and IMMEA for different non-isotropic scattering MIMO V2V scenarios. It is clear that the IMMEA outperforms the MMEA1 and MMEA2 for different non-isotropic scattering MIMO V2V scenarios. Comparing the MMEA1 and MMEA2, we can observe that neither of them consistently 


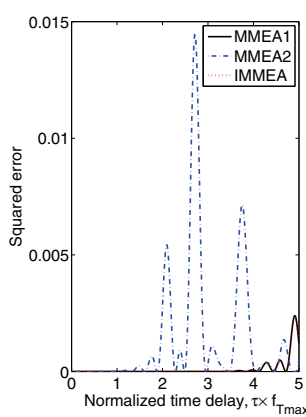

(a)

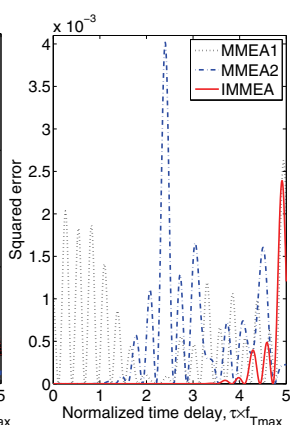

(b)

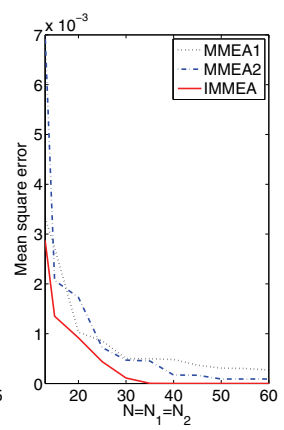

Fig. 1. Squared error $\varepsilon$ of the STCFs for different non-isotropic scattering MIMO V2V channels with $\delta_{T}=\delta_{R}=0.5 \lambda$ : (a) $\mu_{T}=\mu_{R}=0^{\circ}$ and (b) $\mu_{T}=\mu_{R}=50^{\circ}$, and (c) MSE $\bar{\varepsilon}$ of the STCFs for a non-isotropic scattering MIMO V2V channel with $\delta_{T}=\delta_{R}=0.5 \lambda$ and $\mu_{T}=\mu_{R}=50^{\circ}$.

outperforms the other for all non-isotropic scattering MIMO V2V scenarios. This is due to the fact that neither the MMEA1 or MMEA2 has the ability to meet Criterion 2 for all nonisotropic scattering MIMO V2V scenarios as addressed in Section III. Since the IMMEA will reduce to the MMEA1 when $\mu_{T}=\mu_{R}=0^{\circ}$, it is not surprising that the MMEA1 and IMMEA have the same performance in Fig. 1 (a). Fig. 1 (b) also shows that the MMEA1 results in a relatively large squared error $\varepsilon$ even in shorter time delays, which are of most interest for most communication systems [5]. This is because that the MMEA1 designs the AoD/AoA in a non-sufficient range when the mean $\mathrm{AoD} / \mathrm{AoA}$ are larger than zero. In this case, the MMEA1 violates Criterion 1.

Fig. 1 (c) illustrates the mean square error (MSE) $\bar{\varepsilon}$ of the STCF, defined as $\bar{\varepsilon}=\frac{1}{\tau_{\max }} \int_{0}^{\tau_{\max }} \varepsilon d \tau$, with different $N=$ $N_{1}=N_{2}$ for the simulation model by using the MMEA1, MMEA2, and IMMEA for a non-isotropic scattering MIMO $\mathrm{V} 2 \mathrm{~V}$ channel. Here, $\tau_{\max }$ denotes an approximate time interval $\left[0, \tau_{\max }\right]$ over which the approximation of $\rho_{h_{p q} h_{p^{\prime} q^{\prime}}}(\tau)$ is of interest. Fig. 1 (c) clearly shows the convergence behavior of $\widetilde{\rho}_{\widetilde{h}_{p q} \widetilde{h}_{p^{\prime} q^{\prime}}}(\tau) \rightarrow \rho_{h_{p q} h_{p^{\prime} q^{\prime}}}(\tau)$ with the increase of $N$ for all the three methods. The MSE $\bar{\varepsilon}$ for the IMMEA is always smaller than those for the MMEA1 and MMEA2 with the same values of $N$. This indicates that the proposed IMMEA outperforms the MMEA1 and MMEA2 for any values of $N$.

Figs. 2 (a) and (b) show the STCF comparisons of the IMMEA, MMEA1, MMEA2, and reference model under different non-isotropic scattering MIMO V2V scenarios. It is obvious that compared with the MMEA1 and MMEA2, the proposed IMMEA demonstrates a better approximation to the STCF of the reference model with the same complexity $\left(N_{1}=N_{2}=30\right)$. Moreover, due to the violation of Criterion 1, the MMEA1 has poor performance. Comparing Figs. 2 (a) and (b), we find that the increase of the antenna element spacings, i.e., $\delta_{T}$ and $\delta_{R}$, increases the difficulty in approximating the desired STCF of the reference model.

\section{CONCLusions}

In this letter, a new parameter computation method, named as IMMEA, for deterministic SoS simulation models has been proposed under the condition of non-isotropic scattering MIMO V2V Rayleigh fading channels. The proposed IMMEA

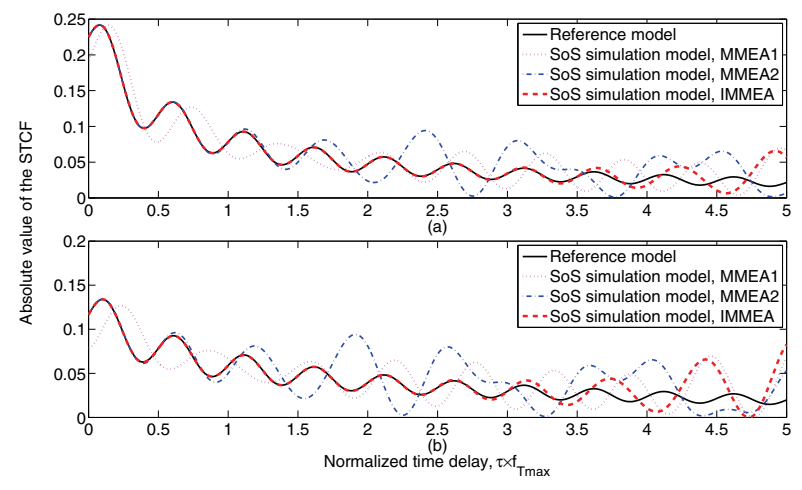

Fig. 2. Absolute values of the STCFs for a non-isotropic scattering MIMO V2V channel with $\mu_{T}=\mu_{R}=50^{\circ}$ and different antenna spacings: (a) $\delta_{T}=$ $\delta_{R}=0.5 \lambda$ and (b) $\delta_{T}=\delta_{R}=\lambda$.

is the first parameter computation method that has the ability to meet the accuracy-efficiency design criteria for all nonisotropic scattering MIMO V2V scenarios. Numerical results have shown that compared with existing MMEAs, the IMMEA provides the same efficiency, while it offers better approximations to the STCF of the reference model.

\section{REFERENCES}

[1] C.-X. Wang, X. Cheng, and D. I. Laurenson, "Vehicle-to-vehicle channel modeling and measurements: recent advances and future challenges," IEEE Commun. Mag., vol. 47, no. 11, pp. 96-103, Nov. 2009.

[2] M. Pätzold, C.-X. Wang, and B. O. Hogstad, "Two new sum-of-sinusoidsbased methods for the efficient generation of multiple uncorrelated Rayleigh fading waveforms," IEEE Trans. Wireless Commun., vol. 8, no. 6, pp. 3122-3131, June 2009.

[3] C. X. Wang, D. Yuan, H. H. Chen, and W. Xu, "An improved deterministic SoS channel simulator for efficient simulation of multiple uncorrelated Rayleigh fading channels," IEEE Trans. Wireless Commun., vol. 7, no. 9, pp. 3307-3311, Sept. 2008.

[4] C. X. Wang, M. Pätzold, and D. Yuan, "Accurate and efficient simulation of multiple uncorrelated Rayleigh fading waveforms," IEEE Trans. Wireless Commun., vol. 6, no. 3, pp. 833-839, Mar. 2007.

[5] C. S. Patel, G. L. Stüber, and T. G. Pratt, "Simulation of Rayleighfaded mobile-to-mobile communication channels," IEEE Trans. Commun., vol. 53, no. 11, pp. 1876-1884, Nov. 2005.

[6] A. G. Zajić and G. L. Stüber, "Space-time correlated mobile-to-mobile channels: modelling and simulation," IEEE Trans. Veh. Technol., vol. 57, no. 2, pp. 715-726, Mar. 2008.

[7] X. Cheng, C.-X. Wang, D. I. Laurenson, S. Salous, and A. V. Vasilakos, "An adaptive geometry-based stochastic model for non-isotropic MIMO mobile-to-mobile channels," IEEE Trans. Wireless Commun., vol. 8, no. 9, pp. 4824-4835, Sept. 2009.

[8] B. O. Hogstad, M. Pätzold, N. Youssef, and D. Kim, "A MIMO mobile-tomobile channel model-part II: the simulation model," IEEE PIMRC'05, Berlin, Germany, Sept., 2005, pp. 562-567.

[9] M. Pätzold, B. O. Hogstad, and N. Youssef, "Modeling, analysis, and simulation of MIMO mobile-to-mobile fading channels," IEEE Trans. Wireless Commun., vol. 7, no. 2, pp. 510-520, Feb. 2008.

[10] A. G. Zajić and G. L. Stüber, "Three-dimensional modeling, simulation, and capacity analysis of space-time correlated mobile-to-mobile channels," IEEE Trans. Veh. Technol., vol. 57, no. 4, pp. 2042-2054, July 2008.

[11] X. Cheng, C.-X. Wang, D. I. Laurenson, S. Salous, and A. V. Vasilakos, "New deterministic and stochastic simulation models for non-isotropic scattering Mobile-to-mobile Rayleigh fading channels," Wireless Commun. Mobile Computing, vol. 11, no. 7, pp. 829-842, July 2011.

[12] M. Pätzold, Mobile Fading Channels. John Wiley \& Sons, 2002.

[13] A. Abdi, J. A. Barger, and M. Kaveh, "A parametric model for the distribution of the angle of arrival and the associated correlation function and power spectrum at the mobile station," IEEE Trans. Veh. Technol., vol. 51, no. 3, pp. 425-434, May 2002. 